\title{
Searching image in blue jays: Facilitation and interference in sequential priming
}

\author{
ALAN B. BOND and ALAN C. KAMIL \\ University of Nebraska, Lincoln, Nebraska
}

\begin{abstract}
Repeated exposure to a single target type (sequential priming) during visual search for multiple cryptic targets commonly improves performance on subsequent presentations of that target. It appears to be an attentional phenomenon, a component of the searching image effect. It has been argued, however, that if searching image is an attentional process, sequential priming should also interfere with performance on subsequent nonprimed targets, and such interference has never been unequivocally demonstrated. In blue jays (Cyanocitta cristata) searching in an operant apparatus for targets derived from images of cryptic moths, detection performance was strongly facilitated in the course of a sequential prime but was relatively unaffected by sequences of mixed target types. Detection accuracy in subsequent probe trials was enhanced by priming with targets of the same type, whereas accuracy on cryptic probes following priming with a more conspicuous target was significantly degraded. The results support an attentional interpretation of searching image.
\end{abstract}

Predators that search for cryptic prey items make use of a wide range of cognitive capabilities. They learn to discriminate the visual features of their prey from the background (Curio, 1976; Krebs, 1973; Lawrence, 1985), they adopt response strategies that maximize their rate of prey discovery (Endler, 1991; Gendron, 1986; Gendron \& Staddon, 1983), and they undergo perceptual changes that temporarily increase their ability to detect more abundant prey types, a phenomenon termed hunting by searching image (Tinbergen, 1960). Tinbergen developed the concept of searching image to account for the fact that insectivorous birds select more abundant prey types disproportionately often, while effectively overlooking rarer ones (Allen, 1988; Bond, 1983; Bond \& Kamil, 1998). Tinbergen noted that his birds tended to take prey items in sequential runs, suggesting that, at any given moment, they were searching for only one kind of prey (see, also, Bond, 1982; Dawkins, 1971). He hypothesized that if the birds were filtering out alternative stimuli and limiting their search to the visual features characteristic of a single prey type, this would increase their ability to detect that prey and reduce the detectability of alternative prey types (Tinbergen, 1960). Over a long series of captures, such a bias could account for the observed population effects (Bond \& Kamil, 1998).

To reproduce the searching image phenomenon in the laboratory and make it accessible to experimental manip-

Our research was supported in part by NSF Grant IBN-9621044. Correspondence concerning this article should be addressed to either A. B. Bond or A. C. Kamil, School of Biological Sciences, 348 Manter Hall, University of Nebraska, Lincoln, NE 68588-0118 (e-mail: abond@ unlserve.unl.edu or akamil@unlserve.unl.edu). Images of moths and cryptic backgrounds, as well as the computer algorithms used in image generation, are available on our Web site (http://niko.unl.edu/ jaylab/virtprey.htm). ulation, Pietrewicz and Kamil $(1977,1979,1981)$ developed an operant analogue, requiring blue jays to search photographic images for either of two cryptically colored moths. Thus, each successive detection trial corresponded to a predation opportunity. When the sequence of prey presentations was modified to produce runs of a single prey type, the birds showed higher levels of accuracy and lower response times than when the two prey types were randomly intermixed. Several other studies have shown a similar improvement in target detectability as a function of stimulus sequence, thereby validating the technique (Blough, 1989, 1991, 1992; Bond \& Riley, 1991; Kono, Reid, \& Kamil, 1998; Langley, 1996; Plaisted \& Mackintosh, 1995).

Tinbergen's (1960) hypothesis has been investigated extensively over the past several decades, at least in part because it appears to provide a compelling context for the operation of attentional processes in visual search. Blough $(1989,1991)$ and Langley $(1996)$ have been particularly forthright in interpreting the facilitation of prey detectability in the Pietrewicz and Kamil (1979) approach as an attentional priming effect, in which a representation of the sought-for target is activated during a block of trials of a single target type. The active representation, it is argued, improves the bird's responsiveness to similar patterns in its visual input and, thereby, facilitates detection of subsequent targets of the same type. Blough (1989) coined the term sequential priming to refer to this technique for eliciting attentional search and has since conducted an extensive series of experiments in which the nature of the sequential priming effect is probed and contrasted with that of more conventional priming techniques (Blough, 1989, 1991, 1992; Blough \& Lacourse, 1994; Vreven \& Blough, 1998).

Plaisted (1997; Plaisted \& Mackintosh, 1995) has recently challenged the attentional account of searching 
image, basing her criticism on a limited capacity model of attention (Broadbent, 1958, 1971; Kahneman, 1973). According to this model, an attentional search could increase the detectability of a given target only at the cost of some reduction in performance on other targets. Plaisted noted that although there was substantial evidence of facilitation of detectability in searching image studies, there had been no correspondingly strong, unequivocal demonstration of interference effects. She concluded that the existing data failed to justify an attentional account and went on to develop an alternative model based on the influence of varying reinforcement rates on the decay of short-term memory. Using pigeons searching for two distinctive checkerboard patterns, Plaisted found evidence that searching was facilitated by reducing the time since the last stimulus of the same type (the interstimulus interval) but that there were no interference effects when the two targets were presented in mixed blocks.

Plaisted's (1997) demand for unequivocal evidence of interference may derive from an overly rigorous interpretation of the limited capacity model. Posner and Snyder (1975) found evidence that facilitation and interference effects are separate processes and are not symmetrical. Facilitation appears to occur in many situations in which interference is not observed. Interference is presumed, however, to play a central role in the population-level effects of searching image, in that stabilization of the prey population requires that rare prey types will tend to be overlooked. It is, thus, noteworthy that the searching image literature has produced few demonstrations of interference that stand up to critical scrutiny. In part, this may be because a strong test of the interference hypothesis requires a fairly elaborate experimental design. Unless one were specifically intending to distinguish interference effects from all other possible interpretations, the necessary controls would not have been run.

To show interference, one must demonstrate that the sequential prime has "simultaneously impaired subjects' ability to detect the other target" (Plaisted, 1997, p. 249), which effectively requires that the subject be primed with a sequence of trials of one type and then tested with a set of subsequent probe trials. Thus, studies that do not include probe trials (Blough, 1991; Kono et al., 1998; Pietrewicz \& Kamil, 1979, 1981) cannot resolve the issue. It is also impossible to distinguish facilitation and interference effects when the primed stimulus is presented simultaneously with an alternative target, as is the case for studies of birds feeding on scattered grain (Bond, 1983; Langley, Riley, Bond, \& Goel, 1996; Reid \& Shettleworth, 1992 ) or for studies in which probe trials contain both the primed and the nonprimed target (Blough, 1992; Langley, 1996; Reid \& Shettleworth, 1992).

Bond and Riley (1991) employed an appropriate design, but their evidence of interference effects was statistically marginal. Several other studies have shown significant interference effects in probes primed with a pretrial associative cue (e.g., Blough, 1989; Lamb, 1988), but they did not run the same experiment with sequential primes.
An additional, striking feature of most of the studies that have claimed to show interference is that the effects have been asymmetrical: Priming with a relatively conspicuous target interferes with subsequent detection of a more cryptic probe, but priming with cryptic targets does not appear to impede responses to more conspicuous probes (Blough, 1989, 1992; Bond \& Riley, 1991; Lamb, 1988; Reid \& Shettleworth, 1992). Even under the most favorable interpretation of the results, therefore, priming appears to induce a process that is only moderately selective (Blough, 1992).

In this research, we address the issue of facilitation and interference, using blue jays searching for cryptic moths, an established experimental system that has previously shown large, robust effects of sequential priming (Kono et al., 1998; Pietrewicz \& Kamil, 1977, 1979, 1981). Because searching image effects are only evident with targets that are very difficult to detect (Blough, 1989; Bond, 1983; Bond \& Riley, 1991; Langley et al., 1996), we developed a stimulus array consisting of digitized images of Catocala moths presented against a complex textured background. By manipulating the generative parameters for the background, we were able to control detectability with far greater precision than has ever been possible with naturalistic stimuli and to examine the effects of changes in both detectability and trial sequence. We employed a rigorous and fully counterbalanced design of sequentially primed and unprimed probe trials to test for interference effects, and, as a final analysis, we extracted a measure of interstimulus interval from our trial sequences and tested, to see whether it would provide a superior account of the sequential priming effect, as Plaisted's (1997) results would predict.

\section{METHOD}

\section{Subjects}

The subjects were 6 blue jays (Cyanocitta cristata) that had been captured in the field as nestlings and hand-reared in the laboratory. The subjects had participated in a number of previous operant experiments involving visual search and detection, including experiments on searching image, using photographs of natural stimuli.

\begin{abstract}
Apparatus
The front panel, consisting of a VGA display (Zenith ZCM-1490; $29 \times 21 \mathrm{~cm})$, occupied one end of the operant chamber $(36 \times 39 \times$ $56 \mathrm{~cm})$. The display was covered with a thin sheet of Lexan polycarbonate, mounted on foam pads to provide a resilient pecking surface, and surrounded by an infrared touch screen bezel (Carroll Touch Smart Frame) to record pecks. Rewards, consisting of halves of mealworm (Tenebrio molitor larvae), were delivered by a motorized lazy Susan into a food well centered $11 \mathrm{~cm}$ below the touch screen. Following delivery of a reward, a food light was switched on, illuminating the mealworm from below. A houselight centered above and directed away from the screen provided diffuse ambient illumination, while avoiding screen reflections. Ambient sounds were attenuated with white noise. Data acquisition and device control were handled with a $50-\mathrm{MHz} 80486 \mathrm{PC}$.
\end{abstract}

\footnotetext{
Stimuli

Photographs of three underwing moths - a light and a dark morph of Catocala relicta and a normal morph of Catocala retecta-were
} 


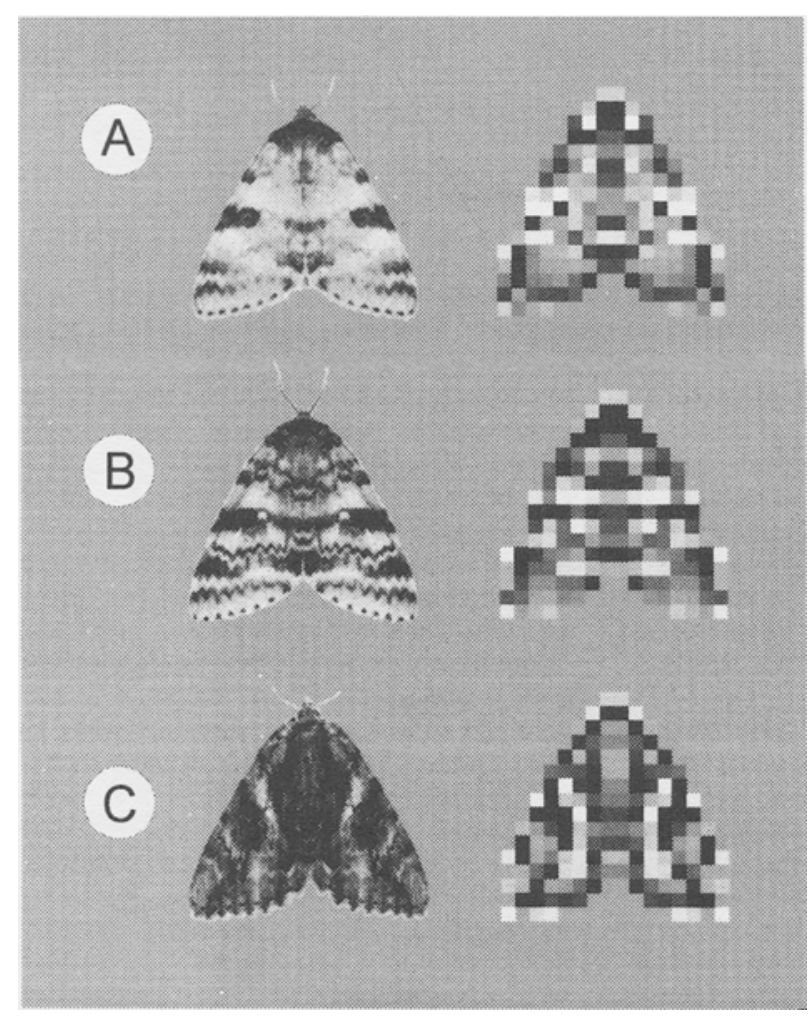

Figure 1. Gray-scale images of the three moth stimuli before and after reduction to 16-pixel squares and histogram normalization. A. Catocala retecta (light morph). B. Catocala retecta (dark morph). C. Catocala relicta.

scanned from plates in Sargent (1976) and reformed into a consistent pose with the head oriented upward and the wings folded, exposing only the cryptic upper surfaces of the forewings. The digital images were then reduced to fit in a 16-pixel square, converted to gray scale at Super VGA resolution $(640 \times 480 \times 64$ gray levels), and histogram normalized to equate the range of intensity values in the three moth types (Figure 1). Corresponding pixels were averaged about the vertical axis to ensure symmetry. Those areas of the 16-pixel square that were not part of the moth were recoded to a blue field value that would register as transparent when the stimulus was displayed on a cryptic background. The target stimuli were, thus, symmetrical, roughly triangular, and filled with a complex texture of gray-scale pixels.

Backgrounds of varying degrees of crypticity were obtained by random sampling of pixel values from a generating distribution. The pooled frequency distribution of gray-scale values from the three moth images was roughly bimodal, suggesting that a bimodal generating distribution would produce backgrounds against which the moths would be maximally difficult to detect. We systematically manipulated the parameters of the generating distribution and evaluated the apparent crypticity of the moth stimuli against the resulting backgrounds. To our eyes, the highest concealing effect was obtained with a bimodal distribution with equal-variance peaks at intensity levels of 6 and 56 (on a scale of $0-63$ ). To vary the task difficulty, we generated images that were random mixtures of pixels from this distribution and another, unimodal distribution with a mode of 30, against which the digital moths appeared to be far more conspicuous. The difficulty level was coded as an integer, with 1 indicating that $10 \%$ of the pixels came from the bimodal distribution, 2 meaning $20 \%, 3$ meaning $30 \%$, and so on. In the final step, a frac- tal texture was imposed on the background, increasing the granularity to correspond more closely to the patterns exhibited by the digital moths (Figure 2).

\section{Trials}

Trials began with presentation of a start key, a red $2.7-\mathrm{cm}$ disk in the center of the screen. A single peck delivered to the start key produced the stimulus configuration: two $9.5 \times 13 \mathrm{~cm}$ fields of background, separated by a 6-cm-wide stripe of uniform gray that contained the advance key, a green $2.7-\mathrm{cm}$ disk. During positive trials, a target was overlaid at a randomly chosen point in the background fields; during negative trials, no target was present. A single peck to the display in the stimulus area blanked the screen and initiated the response outcome. The correct response to a positive trial was to peck the target; the correct response to a negative trial was to peck the advance key. Correct responses to positives were rewarded with pieces of mealworm, followed by an intertrial interval (ITI) of $12 \mathrm{sec}$. Pecks to the advance key, whether correct or incorrect, produced no food reward but reduced the subsequent ITI to $6 \mathrm{sec}$. Pecks to the background were punished with a 30-sec ITI. If the bird failed to make any response to a display for $60 \mathrm{sec}$, the screen was blanked, and the same trial type, but with a new background and target position, was presented again after a $12-\mathrm{sec}$ delay.

\section{Training}

The subjects were initially trained to peck at the target stimuli on a uniform gray background and were then given extensive discrimination training in daily 70 -trial sessions. Discrimination trials were randomly ordered, with each session consisting of an equal number of positives and negatives and with positive trials consisting of equal numbers of the three target types. In each session, equal numbers of backgrounds of four different degrees of difficulty were used, in randomized order. All the subjects began training with backgrounds 1 through 4 , and task difficulty was gradually increased over successive sessions. The goal of training was to obtain a level of difficulty in which all three targets were detected with a probability of .7-.8, aggregated over blocks of 5 consecutive sessions. If performance at the end of a block showed signs of having exceeded criterion, we shifted the bird to the next level of background difficulty and resumed training. Stable performance within the desired accuracy limits was obtained in about 55 sessions for all 6 birds. Four of the subjects stabilized to criterion levels with backgrounds 3 through 6,1 required backgrounds 4 through 7 , and 1 required 6 through 9 .

\section{Testing}

Each experimental session consisted of 64 trials, 32 positives and 32 negatives. Imbedded within each session were two treatment blocks of 16 trials each, 8 positives and 8 negatives. Trials within treatment blocks were block randomized in sets of four, to ensure that no more than 2 positives occurred in succession. Immediately following each treatment block was a 4-trial probe block, consisting of 2 positives of one of the three target types and 2 negatives, in random order. The two treatment blocks, with their associated probes, were separated from one another by 12 buffer trials containing equal numbers of the three target types in random order, intermixed with an equal number of negative trials. Between 5 and 7 additional buffer trials were provided at the beginning and the end of the session.

The positive trials in a treatment block consisted either of a single target type (a run of the given stimulus) or of all three target types (a nonrun). Because the number of positive trials in a block was not evenly divisible by three, it was not possible to display exactly equal numbers of the three targets in each nonrun block. The minority target was, however, varied systematically across sessions, to ensure that all three targets were equally abundant across all nonrun blocks. Over the course of 90 experimental sessions, each bird was exposed 


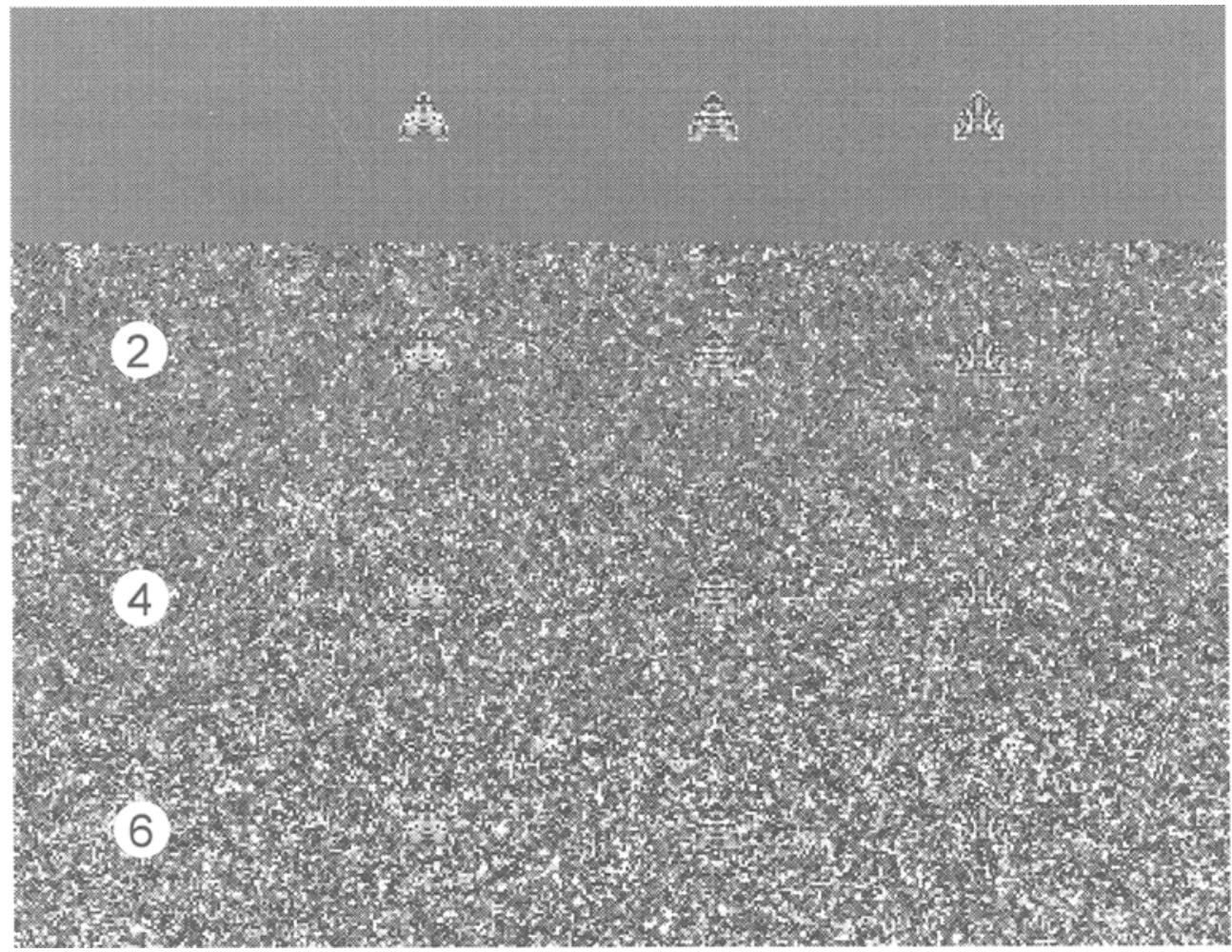

Figure 2. Three digital target types displayed on backgrounds of three levels of crypticity (levels 2,4 , and 6). The prey types are (left to right) Catocala retecta (light morph), Catocala retecta (dark morph), and Catocala relicta.

to 180 treatment blocks, 45 of each of the three types of run blocks and 45 nonrun blocks, followed by each of the three types of probe blocks. The design was fully counterbalanced within subjects, with the constraint that the 2 treatment blocks within a single session were never runs of the same target type.

\section{Analysis}

For the analysis of background and stimulus sequence effects, we determined the length of the immediately previous run of correctly detected positives of a single target type for each trial in the run blocks. As an independent variable, the number of prior detected targets is more sensitive than is simply using the serial position in the run block, since it adjusts for the actual prior experience of the subject (Kono et al., 1998). We reduced the measure to a three-level category variable, grouping together trials with zero or one previous detection, trials with two to four previous detections, and trials with five or more. To provide a control for the effects of repeated rewards, we extracted an analogous measure, the number of previous correctly detected targets of any type, from the nonrun blocks. The background difficulty variable was recoded to enable comparisons across subjects, with 1 indicating the lowest crypticity experienced by that subject and 4 indicating the highest.

Accuracy was estimated by calculating the percent correct responses for each combination of bird, stimulus type, background, and number of previous detections. To reduce ceiling effects, the accuracy values were subjected to an arcsine transform (Sokal \& Rohlf, 1981). Response time was estimated as the mean survival time within subjects, using Greenwood's formula and the productlimit method (Kalbfleisch \& Prentice, 1980), for each combination of independent variables (SAS LifeTest Procedure; SAS, 1990). For the analysis of probe effects, we extracted the first positive trial from each probe block and categorized it according to probe stim- ulus type and run treatment type. There were, thus, 15 probe trials at each of 12 treatment combinations ( 3 probes $\times 4$ run treatments) for each bird. Accuracy and response time were estimated within subjects for each treatment combination, as in the sequence analysis.

To estimate the effects of interstimulus interval (Blough, 1991; Plaisted, 1997) on accuracy and response time for positive trials, each positive trial was categorized by stimulus type and number of previous detections, as well as by the elapsed time since the offset of the last previous correctly detected trial of the same type. This measure of the interstimulus interval thus incorporated ITIs and the response times to intervening negative trials and erroneously rejected positive trials. It ranged from a minimum of $12 \mathrm{sec}$ to a maximum of nearly $20 \mathrm{~min}$.

When the interstimulus interval was categorized into 30 -sec bins and compared with the distribution of the number of previous detections, it was evident that virtually all of the intervals over $3 \mathrm{~min}$ were from trials in which there was zero or one prior detection of the given stimulus. To compare the predictive effectiveness of the two measures, we therefore restricted the interstimulus interval to three categories: less than $30 \mathrm{sec}$, between $30 \mathrm{sec}$ and $1 \mathrm{~min}$, and between 1 and $3 \mathrm{~min}$. All the trials with longer intervals were discarded. The final data set consisted of 10,721 trials ( $31 \%$ of the total), distributed roughly equally across the three categories. For this reduced set of positive stimuli, we calculated accuracy and mean response time within bird, stimulus type, and treatment category.

\section{RESULTS}

\section{Background Effects}

Response accuracy on negative trials was uniformly high, averaging $95 \%$ correct across all subjects and back- 


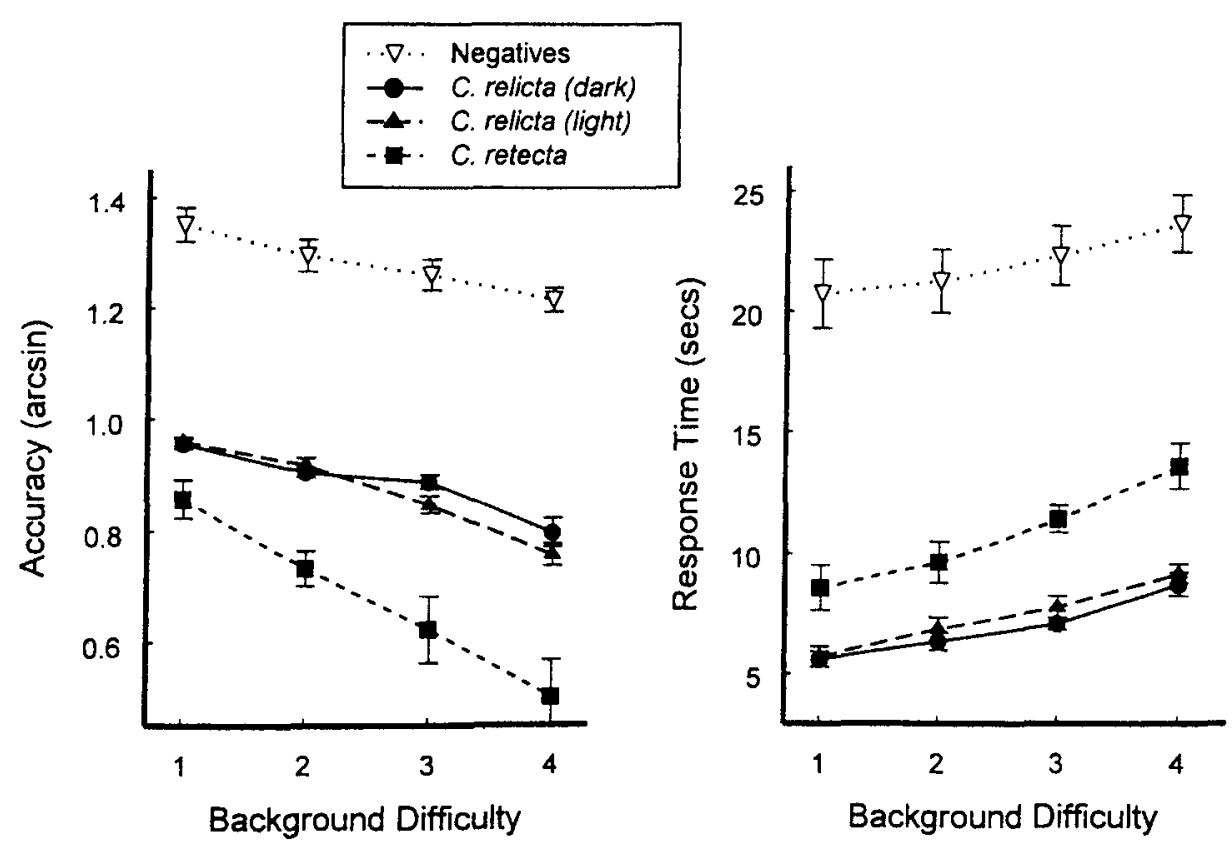

Figure 3. Effects of background difficulty on mean accuracy (left panel) and response time (right panel), plotted separately for the three target types and negatives. Error bars indicate variation across subjects within treatments $( \pm 1 S E)$.

grounds. Positive trial accuracy was somewhat lower, averaging $78 \%$ correct. Misses, in which the bird erroneously pecked the advance key, constituted most of the errors to positive stimuli. Only $2.5 \%$ of the responses to positive stimuli were false positives-that is, pecks to the background away from the overlaid target. Correct response time to negatives was nearly three times as long as that to positives $(21.9 \mathrm{vs} .7 .9 \mathrm{sec})$, consistent with an exhaustive search of the display. Accuracy decreased and response time increased with increasing levels of background difficulty for both positive and negative trials (Figure 3 ). A repeated measures analysis of variance (ANOVA) on positive trials showed significant effects of background on accuracy $[F(3,15)=13.0]$ and response time $[F(3,15)=14.0]$. Similar outcomes were obtained for negatives [accuracy: $F(3,15)=26.9$; response time: $F(3,15)=$ 5.89], confirming the success of our efforts to manipulate crypticity.

As in earlier operant studies (Bond \& Riley, 1991), accuracy and response time were negatively correlated even within detectability and sequence treatments. A plot of accuracy against response time for each of the four levels of background difficulty for trials with zero or one previous detection produced four parallel lines with negative slopes (Figure 4). An analysis of slope heterogeneity, using a repeated measures analysis of covariance, confirmed that the slope was significantly less than zero $[F(1,5)=71.9]$ but that there were no significant slope differences between background levels $[F(3,15)<1]$. There were clear effects on the intercept parameter, however:
The intercept for background level 1 was significantly higher than that for background level $4[t(10)=2.24]$.

\section{Sequence Effects}

A three-way repeated measures ANOVA (target type $\times$ background $\times$ previous detections) on positive trials found a significant main effect of target type for both accuracy $[F(2,10)=13.8]$ and response time $[F(2,10)=$ 12.6; Figure 5A]. Subsequent contrast analysis indicated that responses to $C$. retecta were significantly slower $[F(1,5) \geq 10.7]$ and less accurate $[F(1,5) \geq 10.5]$ than those to either of the two morphs of $C$. relicta. Differences between the two relicta morphs were much smaller. Changes in background clearly had a larger impact on detection of the light morph than of the dark one (Figure 3), however, suggesting that the dark form was generally more conspicuous. The main effect of previous experience was also significant: Accuracy increased $[F(2,10)=$ $28.9]$ and response time decreased $[F(2,10)=5.97]$ with increasing number of prior detections (Figure $5 \mathrm{~A}$ ). The only significant interaction was the effect of background $\times$ previous detections for accuracy $[F(6,30)=2.57]$, apparently resulting from an enhanced effect of previous detections on more cryptic backgrounds.

The same three-way repeated measures ANOVA applied to the nonruns trials yielded similar main effects of target type on accuracy $[F(2,10)=19.6]$ and response time $[F(2,10)=7.85$; Figure $5 \mathrm{~B}]$. There was also a significant main effect of number of previous detections on accuracy $[F(2,10)=5.84]$, although no significant effect 


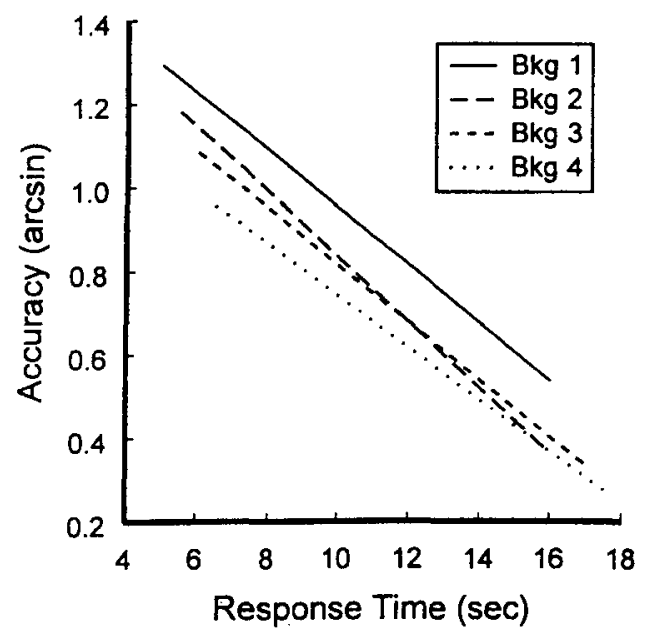

Figure 4. Regression lines displaying the relationship between accuracy and response time for four levels of background difficulty. All the points were from trials with zero or one previous detection of the stimulus.

on response time was observed $[F(2,10)<1]$. As in the analysis of run trials, the only significant interaction was the effect of background $\times$ previous detections for accuracy $[F(6,30)=3.54]$.

To determine whether sequential priming in the run blocks had a significant impact on accuracy beyond the effects of repeated detections, we conducted a four-way, repeated measures ANOVA (treatment [i.e., run vs. nonrun] $\times$ target type $\times$ background $\times$ previous detections) on the combined data from all the treatment blocks. The primary effect of interest was the interaction of treatment with number of previous detections, which was significant $[F(2,9)=4.07]$, establishing that repeated detections of a single target type did, in fact, improve accuracy above the levels that resulted from a series of detections of mixed target types. The magnitude of the difference between treatment and control was substantial (Figure 5A vs. 5B). Accuracy increased by an average of $19.8 \%$ over the course of a run block, but only by $4.3 \%$ over a nonrun block. Response time decreased by $11.0 \%$ over the course of a run block but increased by an average of $7.6 \%$ over a nonrun block (this latter result was not statistically significant).

A three-way ANOVA (prior target type $\times$ background $\times$ number of previous detections) on negative trials from run blocks showed no effects on accuracy but significant main effects on response time of prior target type $[F(2,10)=6.49]$ and previous detections $[F(2,10)=6.32]$, as well as a significant interaction $[F(4,18)=3.20]$. A subsequent contrast analysis suggested that both main effects were meaningful. Responses to negatives following $C$. retecta trials were slower than those following other target types [23.05 vs. $21.66 \mathrm{sec} ; F(1,10)=14.6$ ], and this was true even following only a single positive trial [21.63 vs. $22.69 \mathrm{sec} ; F(1,10)=11.0]$. Responses to negatives following runs of five or more of the same tar- get type were also slowed, relative to those following a shorter series of positives [ 22.87 vs. $21.75 \mathrm{sec} ; F(1,10)=$ $8.45]$. The significant interaction appeared to reflect the fact that the previous detection effect was evident for only two of the three targets.

\section{Probe Effects}

To test for probe effects, we categorized the run treatment for each probe trial as (1) a nonrun treatment (Control), (2) a sequential prime of the same target type (Same), or (3) a prime of a different target type (Diff). A two-way repeated measures ANOVA (treatment type $X$ probe target type) showed significant main effects of run treatment on accuracy $[F(2,10)=11.3]$ and of probe target type on accuracy $[F(2,10)=13.7]$ and response time $[F(2,10)=5.27]$. None of the interactions was significant. Subsequent planned contrasts showed that the effect of run treatment on accuracy was due to more accurate responding following Same primes than following either Controls $[F(1,10)=11.1]$ or Diff primes $[F(1,10)=21.2]$.

Accuracy on probe trials following Diff primes was not significantly lower than that following Controls $[F(1,10)=1.59]$, implying no interference effect from prior experience with a different target type. However, several other studies (Blough, 1989, 1992; Bond \& Riley, 1991; Reid \& Shettleworth, 1992) have noted that interference effects in visual search are often asymmetrical: Priming with targets that are more conspicuous than the probe may interfere with probe detection, whereas priming with less conspicuous targets may not. To test this possibility, we recategorized different prime treatments according to whether the target type in the priming run was more conspicuous (Diff-Conspicuous) or more cryptic (Diff-Cryptic) than the probe target.

This recategorization assorted trials unequally across probe types. All Diff treatments for the most cryptic probe type were Diff-Conspicuous, whereas all Diff treatments for the most conspicuous probe type were Diff-Cryptic. To avoid confounding the results with the significant main effect of probe type, we transformed accuracy and response time for each subject, probe type, and run treatment to the difference from the corresponding Control value. A repeated measures ANOVA on the accuracy and response time difference scores by treatment type (Same vs. Diff-Cryptic vs. Diff-Conspicuous) showed a significant effect of treatment on accuracy $[F(2,10)=17.5]$, but no effect on response time (Figure 6). Subsequent contrast analysis showed that Same treatments produced higher relative accuracy than did either type of Diff treatment $[F(1,10) \geq 5.75]$ and that Diff-Conspicuous treatments resulted in lower accuracy, relative to controls, than did Diff-Cryptic treatments $[F(1,10)=12.2]$. The relative effect of Diff-Cryptic treatments was, in fact, not significantly different from zero $[t(5)=1.12]$.

\section{Effects of Interstimulus Interval}

If Plaisted's (1997) assertions concerning the causal primacy of interstimulus interval in visual search for 
A

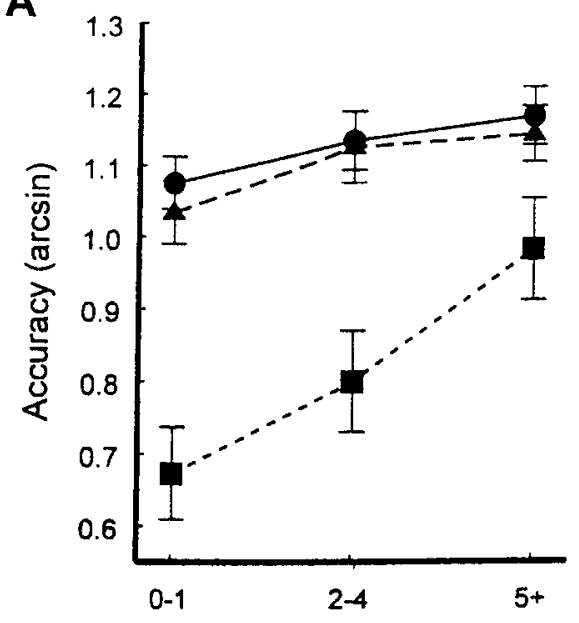

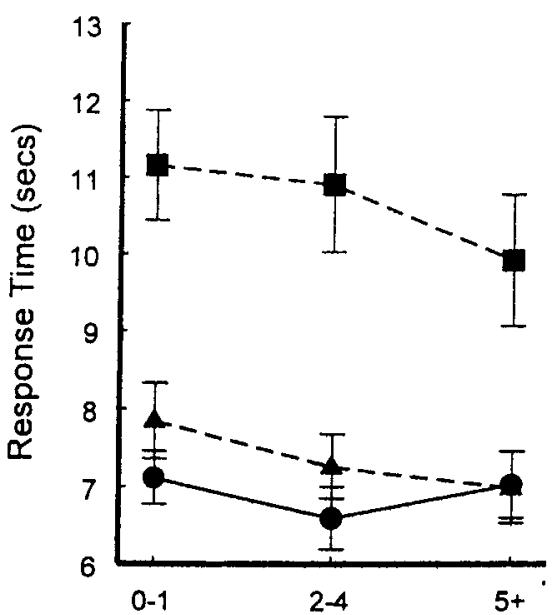

Number of Prior Detections
B

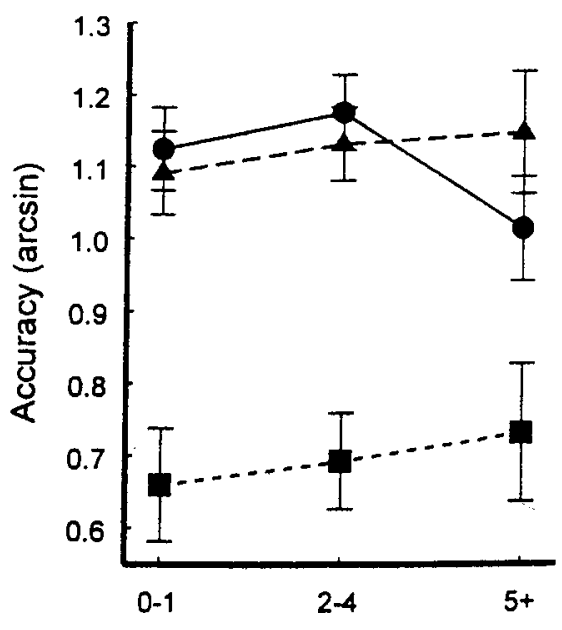

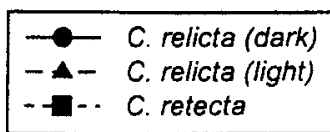

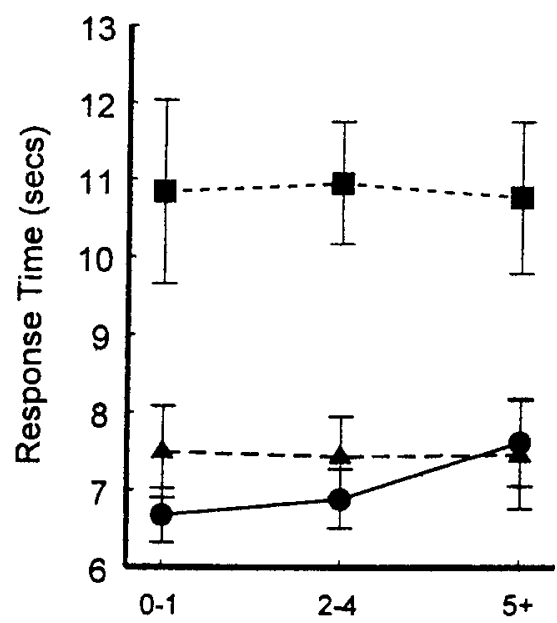

Number of Prior Detections

Figure 5. A. Effect of number of previous detections on mean accuracy (left panel) and response time (right panel) for trials in run blocks, plotted separately by target type. B. Effect of previous detections on mean accuracy (left panel) and response time (right panel) for trials in nonrun blocks. Error bars indicate variation across subjects within treatments $( \pm 1 S E)$.

cryptic targets are valid, we should see certain predictable effects of the time interval on detection performance. In particular, if the effects of interstimulus interval and previous detections are assessed independently, accuracy should decrease and response time increase with increases in the interstimulus interval, and the magnitude of the treatment effect ( $\omega^{2}$; Keppel, 1982) should be higher for the interstimulus interval than for the number of previous detections. In addition, any apparent effect of previous detections should simply be a reflection of its relationship to the interstimulus interval. If, therefore, we include both measures in the same ANOVA model, and if we use Type IV, or partial, sums of squares in the combined analysis, the common variance owing to the relationship between the two measures will be factored out (SAS, 1990), and, by Plaisted's hypothesis, the main effect of number of previous detections should disappear.

Repeated measures ANOVAs were conducted, using either target type and interstimulus interval or target type and number of previous detections as predictors. The main effect of interstimulus interval on accuracy was not significant $[F(2,10)=2.00]$, but there was a significant 


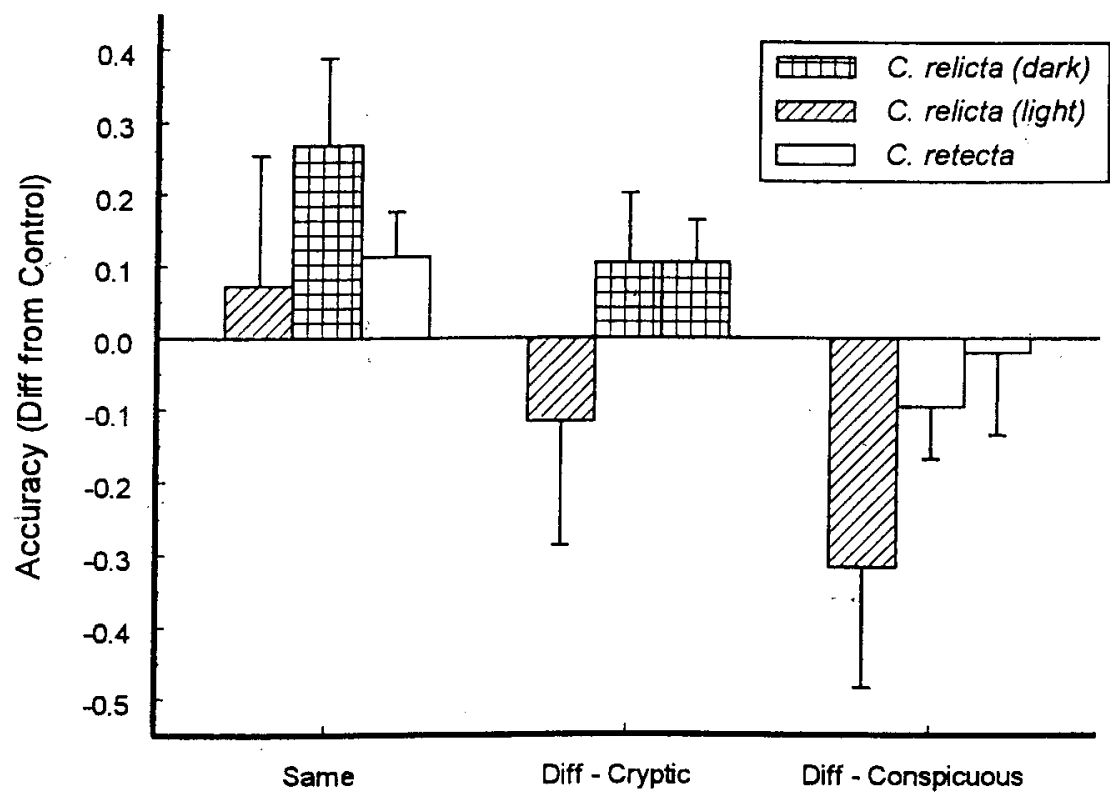

Run Treatment Type

\begin{abstract}
Figure 6. Effects on accuracy of responses to probe trials, in terms of difference from control (nonrun) treatment. Grouping variable for bars is treatment type: Same (run block of same target type as probe), Diff-Cryptic (target in run block more cryptic than target in probe), or Diff-Conspicuous (target in run block less cryptic than target in probe). Target type for probe is indicated by the bar pattern, as shown in the figure legend. Error bars indicate variation across subjects within treatments $( \pm 1 S E)$.
\end{abstract}

main effect on response time $[F(2,10)=5.27$; Figure $7 \mathrm{~B}]$. In contrast, the main effect of previous detections on accuracy was highly significant $[F(2,10)=8.44]$, but there was no effect, in this reduced data set, on response time $[F(2,10)=1.63$; Figure $7 \mathrm{~A}]$. The magnitude of the treatment effects $\left(\omega^{2}\right)$ was about 0.02 for both accuracy and response time for previous detections, and comparable values were obtained for response time for interstimulus interval.

The direction of the effects of interstimulus interval was, however, opposite to that predicted by Plaisted's (1997) hypothesis. Although accuracy increased with increasing previous detections, as was observed in previous analyses (Figure $5 \mathrm{~A}$ ), the main effect of interstimulus interval on response time resulted from a decrease in response time with longer intervals, not from the increase Plaisted would have predicted. There was an additional suggestion that accuracy may have increased with increasing interstimulus intervals, at least for C. retecta (Figure 7B), which would also be in the opposite direction from that predicted on the basis of Plaisted's hypothesis.

Combining the two measures in one three-way repeated measures ANOVA had little impact on the observed effects. As in the single-variable analyses, previous detections had a significant main effect on accuracy $[F(2,10)=10.1]$, but no effect on response time $[F(2,10)<1]$, whereas interstimulus interval had a significant effect on response time $[F(2,10)=4.40]$, but no effect on accuracy $[F(2,10)=$ 1.73]. Contrary to Plaisted's (1997) hypothesis, therefore, inclusion of the interstimulus interval in the analysis did not remove the main effect of the number of previous detections, indicating that differences in interstimulus interval were not the primary source of the facilitation.

\section{DISCUSSION}

The results of this study provide striking evidence of facilitation of detection, in terms of both accuracy and response time, following a sequential prime. The improvement was apparent even for relatively conspicuous targets but was most impressive in the case of $C$. relicta, the most cryptic of the three moths. Detection accuracy for this species was over $50 \%$ greater in trials following five or more positives of the same type than in those following one or fewer (Figure 5A). The magnitude of the treatment effect produced by sequential priming was comparable with that resulting from differences in background crypticity. Unlike earlier studies on pigeons (Blough, 1989, 1992), there was an interaction between priming and detectability, with larger priming effects being exhibited on more difficult backgrounds. Response time decreases were more modest, on the order of $10 \%-15 \%$, and were apparently of similar magnitude for all three targets (Figure 5A). Blough (1989) has remarked 
A

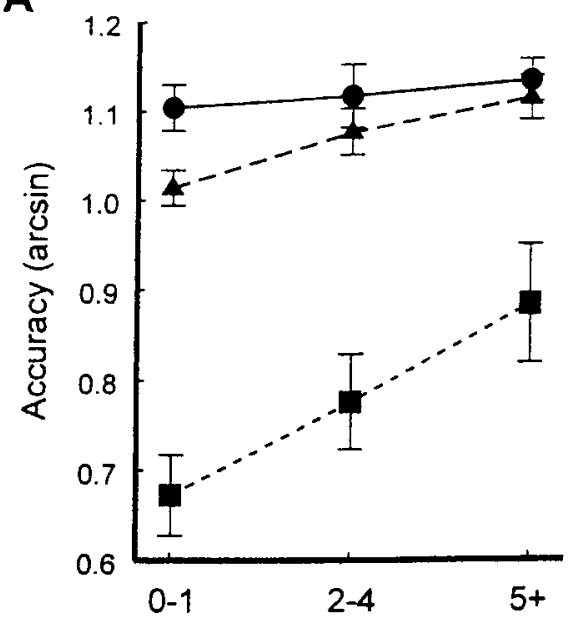

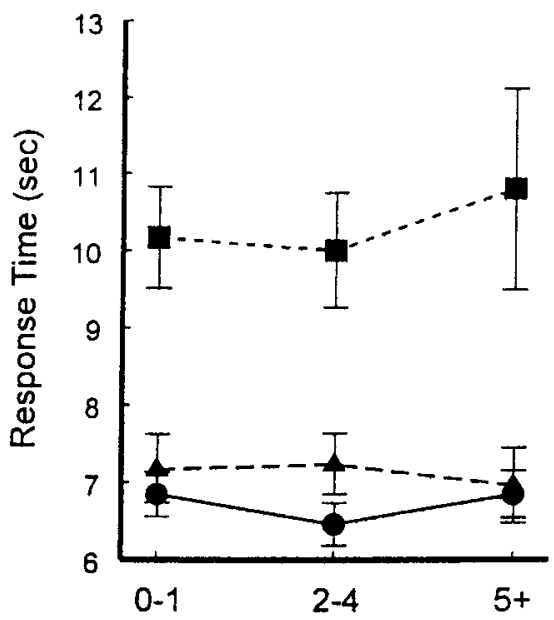

Number of Prior Detections

B
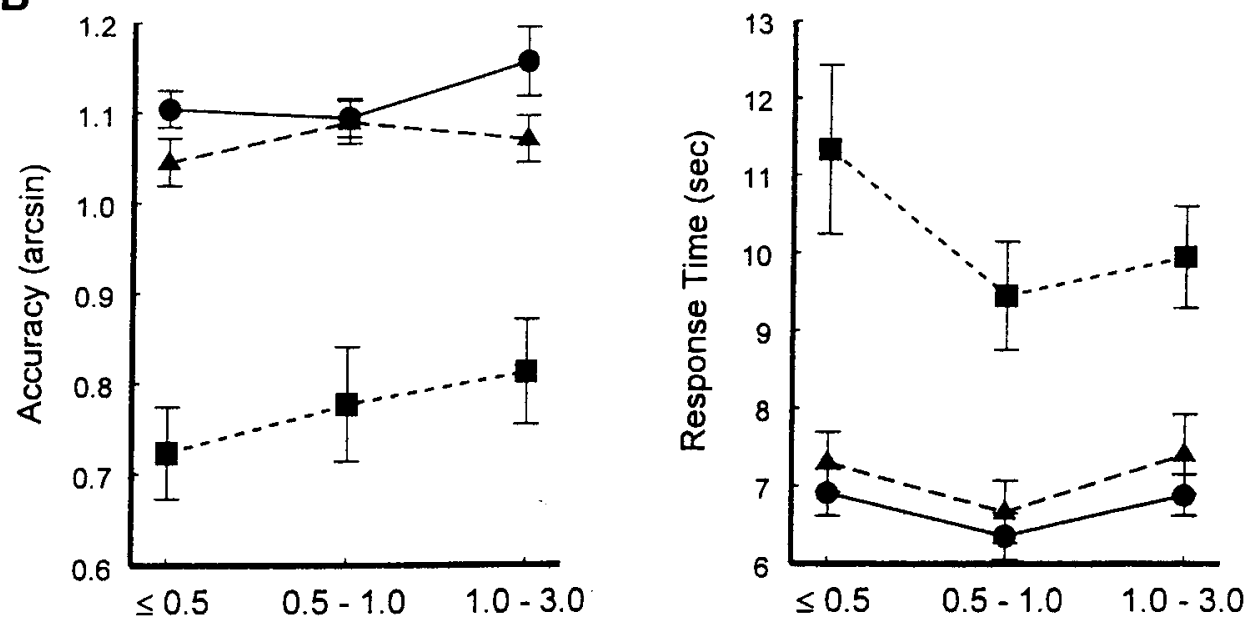

Interstimulus Interval ( $\mathrm{min})$

Figure 7. (A) Effects of number of previous detections on accuracy (left panel) and response time (right panel) for the reduced, comparison data set, plotted by target type. (B) Effects of interstimulus interval on accuracy (left panel) and response time (right panel) for the comparison data set, plotted by target type. Error bars indicate variation across subjects within treatments $( \pm 1 S E)$.

that more cryptic conditions appear to favor an accuracy effect, and this is consistent with our results.

Performance on negatives, in contrast, was relatively unaffected by sequence manipulations. Accuracy on negative trials was virtually at ceiling across all levels of the number of previous detections, and response time actually increased by about $5 \%$ following runs of five or more positives of the same type. In their study of visual search by blue jays, Pietrewicz and Kamil (1981) found significant effects of trial sequence on the accuracy of responding to negatives, although their initial level of accuracy was not as high. Similarly high accuracy and minimal treatment effects in negative trials were seen in pigeons searching for images of cryptic grain (Bond \& Riley, 1991), suggesting that the results for negative trials in the present study may reflect similar features in the reward regimen, particularly the high premium placed on avoidance of false positives.

Detection accuracy of targets in probe trials was reduced following a sequential prime for a different target type, at least when the probe target was more cryptic than the primed target (Figure 6). This result qualifies as a true 
interference effect: Accuracy on cryptic probes following sequential priming with more conspicuous targets was significantly worse than that following a nonpriming, control block, whereas accuracy on probes of any type was improved above the control level by sequential priming with targets of the same type. Thus, our results appear to satisfy Plaisted's (1997) requirement and to provide clear evidence in favor of an attentional account.

Detection of relatively conspicuous probes was not significantly impacted by a prior sequential prime. Blough (1992) pointed out that such asymmetrical interference effects are not, of themselves, inconsistent with an attentional account of searching image and concluded that the attentional state induced by sequential priming is relatively porous, providing little interference to the detection of salient, nonprimed items. Asymmetrical effects are pervasive in searching image studies, however, even when all the target types are very difficult to detect and when the differences between them in crypticity are small (Blough, 1989, 1992; Bond \& Riley, 1991; Reid \& Shettleworth, 1992). Langley (1996) provides the only evidence of fully symmetrical interference, but she manipulated her digital grain images extensively before testing, to ensure that the two target types would be equally cryptic. The generality of asymmetrical effects in the absence of such careful stimulus manipulation suggests that a common underlying mechanism may be responsible.

Bond and Riley (1991) noted that the direction of the asymmetry in interference effects was consistent with a change in the persistence with which the subjects searched the display. A run of successfully detected, conspicuous positives could encourage the birds to reduce their givingup time for the display, increasing the likelihood of subsequently discarding a cryptic probe prior to the discovery of the target. A run of cryptic targets, on the other hand, might not reduce the giving-up time to the same degree and would, thus, have less of an impact on subsequent discovery of a relatively conspicuous probe. The mechanism is analogous to the scan rate hypothesis proposed by Guilford and Dawkins (1987) as an alternative explanation for searching image effects.

There is no ready substantiation for this hypothesis in the present results, however. The response time to negatives, which one would expect to provide an indication of giving-up time for a display, did not decrease as a result of a run of positives. Negative response times probably also contain components that reflect the bird's recent history of reward for searching the display, a factor that is implicated in the significant main effect of number of prior detections on nonrun positives (Figure 5B). Such anomalous effects, in combination with the extraordinarily long response times observed for negative trials, suggest that responses to the advance key are fairly aversive (Kamil, Misthal, \& Stephens, 1993). There is, thus, no direct, uncontaminated measure of the bird's predisposition to scan the display and no feasible test of the Bond and Riley (1991) hypothesis.
Using pigeons searching for symmetrical checkerboard patterns, Plaisted (1997) found that reducing the interstimulus interval enhanced detection performance, even when two target types were presented in intermixed trials. Indeed, she found no additional benefit resulting from runs of a single target type that could not be accounted for by reductions in interstimulus interval and no indication of interference in nonrun blocks of mixed targets. Our results do not support Plaisted's model. Within the range of interstimulus intervals from $30 \mathrm{sec}$ to 3 min, which are actually slightly longer than those Plaisted used, there was no indication that the interval measure provided a superior explanation of the sequential priming effect. In fact, interstimulus interval had no impact on accuracy, and its effect on response time was inconsistent with Plaisted's prediction (Figure 7). It is possible that Plaisted's results were conditioned by the stimuli she used, which do not seem to be particularly cryptic (see Plaisted \& Mackintosh, 1995), or by her use of only a single pair of target types. Vreven and Blough (1998) have shown that, for their relatively conspicuous stimuli, very experienced pigeons show sequential priming effects only with a set of eight targets, and they are perfectly capable of searching for a set of six targets simultaneously, with no evident impairment of performance.

Our failure to replicate Plaisted's results should not be seen as a denial of the possibility of a temporal decay in the effects of searching image. Indeed, any mechanism that produces a bias in the outcome of visual search for cryptic prey, whether based in short-term memory or selective attention, should fade out over time in the absence of additional discoveries of the sought-for stimulus. Bond (1983) notes that apostatic selection, the presumed ecological effect of hunting by searching image, effectively entails that searching images be discarded after a sufficient duration of unrewarded searching, and apostatic selection has been demonstrated using our system of blue jays and digital moths (Bond \& Kamil, 1998). The lack of temporal effects in the present results may simply reflect a relatively long time course for the decay of a primed representation. Most of the trials we analyzed for interstimulus interval effects occurred at intervals of less than $3 \mathrm{~min}$ from the last previous detection of the same prey type, and 3 min may be close to the minimum duration necessary to demonstrate a temporal decline (Blough \& Lacourse, 1994; Langley et al., 1996). A clearer understanding of the persistence of searching images will require a parametric study in which interstimulus interval is explicitly manipulated over a much broader range.

\section{REFERENCES}

Allen, J. A. (1988). Frequency-dependent selection by predators. Philosophical Transactions of the Royal Society of London: Series B. 319, 485-503.

Blough, P. M. (1989). Attentional priming and search images in pigeons. Journal of Experimental Psychology: Animal Behavior Processes, 15, 211-223. 
Blough. P. M. (1991). Selective attention and search images in pigeons. Journal of Experimental Psychology: Animal Behavior Processes, 17, 292-298

BLough. P. M. (1992). Detectability and choice during visual search: Joint effects of sequential priming and discriminability. Animal Learning \& Behavior, 20, 293-300.

Blough, P. M., \& Lacourse, D. M. ( 1994). Sequential priming in visual search: Contributions of stimulus-driven facilitation and learned expectancies. Animal Learning \& Behavior, 22, 275-281.

BonD, A. B. (1982). The bead game: Response strategies in free assortment. Human Factors, 24, 101-110.

Bond. A. B. (1983). Visual search and selection of natural stimuli in the pigeon: The attention threshold hypothesis. Journal of Experimental Psychology: Animal Behavior Processes, 9, 292.306.

BOND, A. B., \& KAMIL. A. C. (1998). Apostatic selection by blue jays produces balanced polymorphism in virtual prey. Nature, 395, 594596.

Bond, A. B., \& RiLeY. D. A. (1991). Searching image in the pigeon: A test of three hypothetical mechanisms. Ethologv, 87, 203-224.

Broadbent, D. E. (1958). Perception and communication. London: Pergamon.

Broadbent. D. E. (1971). Decision and stress. New York: Academic Press.

Curio, H. (1976). The ethology of predation. Berlin: Springer-Verlag.

Dawkins, M. (1971). Shifts of 'attention' in chicks during feeding. Animal Behaviour, 19, 575-582.

ENDLER, J. A. (1991). Interactions between predators and prey. In J. R. Krebs \& N. B. Davies (Eds.). Behavioural ecology: An evolutionary approach (pp. 169-196). Oxford: Blackwell.

GenDRon, R. P. (1986). Searching for cryptic prey: Evidence for optimal search rates and the formation of search images in quail. Animal Behaviour. 34, 898-912.

Gendron, R. P.. \& Staddon, J. E. R. (1983). Searching for cryptic prey: The effect of search rate. American Naturalist, 121, 172-186.

GuILFORD, T., \& DAwkins, M. S. (1987). Search images not proven: A reappraisal of recent evidence. Animal Behaviour, 35, 1838-1845.

Kahneman, D. (1973). Attention and effort. Englewood Cliffs, NJ: Prentice-Hall.

Kalbfleisch. J. D.. \& Prentice, R. L. (1980). The statistical analysis of failure time data. New York: Wiley.

Kamil, A. C.. Misthal, R. L., \& Stephens, D. W. (1993). Failure of simple optimal foraging models to predict residence time when patch quality is uncertain. Behavioral Ecologv, 4, 350-363.

KEPPEL, G. (1982). Design and analvsis (2nd ed.). Englewood Cliffs, NJ: Prentice-Hall.

KonO, H.. ReID. P. J.. \& KamIL. A. C. (1998). The effect of background cuing on prey detection. Animal Behaviour, 56, 963-992.

KREBS, J. R. (1973). Behavioral aspects of predation. In P. P. G. Bateson \& P. H. Klopfer (Eds.), Perspectives in ethologv (pp. 73-111). New York: Plenum.
LAMB. M. R. (1988). Selective attention: Effects of cuing on the processing of different types of compound stimuli. Journal of Experimental Psychology: Animal Behavior Processes, 14, 96-104.

LANGLEY, C. M. (1996). Search images: Selective attention to specific visual features of prey. Journal of Experimental Psychology: Animal Behavior Processes, 22, 152-163.

Langley, C. M., Riley, D. A., Bond, A. B., \& Goel, N. (1996). Visual search for natural grains in pigeons: Search images and selective attention. Journal of Experimental Psychologv: Animal Behavior Processes, 22, 139-151.

LAWRENCE, E. S. (1985). Evidence for search image in blackbirds (Turdus merula L.): Long-term learning. Animal Behaviour, 33, 1301. 1309.

Pietrewicz, A. T.. \& Kamil, A. C. (1977). Visual detection of cryptic prey by blue jays (Cyanocitta cristata). Science, 195, 580-582.

Pietrewicz, A. T., \& Kamil, A. C. (1979). Search image formation in the blue jay (Cyanocitta cristata). Science, 204, 1332-1333.

Pietrewicz, A. T., \& Kamil, A. C. (1981). Search images and the detection of cryptic prey: An operant approach. In A. C. Kamil \& T. D. Sargent (Eds.), Foraging behavior: Ecological, ethological, and psychological approaches (pp. 311-331). New York: Garland.

PLaisted, K. [C.] (1997). The effect of interstimulus interval on the discrimination of cryptic targets. Journal of Experimental Psychology: Animal Behavior Processes, 23, 248-259.

Plaisted, K. C., \& Mackintosh, N. J. ( 1995). Visual search for cryptic stimuli in pigeons: Implications for the search image and search rate hypotheses. Animal Behaviour, 50, 1219-1232.

Posner, M. I., \& SNYDER. C. R. R. (1975). Facilitation and inhibition in the processing of signals. In P. M. A. Rabbitt \& S. Dornic (Eds.), Attention and performance $V$ (pp. 669-682). San Diego: Academic Press.

Reid. P. J.. \& Shettleworth, S. J. (1992). Detection of cryptic prey: Search image or search rate? Journal of Experimental Psychology: Animal Behavior Processes, 18, 273-286.

SARGENT, T. D. (1976). Legion of night: The underwing moths. Amherst, MA: University of Massachusetts Press.

SAS (1990). SAS/STAT user's guide: Version 6 (4th ed., Vol. 2). Cary, NC: SAS Institute.

Sokal, R. R., \& Rohlf, F. J. (1981). Biometry (2nd ed.). New York: Freeman.

Tinbergen, L. (1960). The natural control of insects in pine woods: I. Factors influencing the intensity of predation by songbirds. Archives Néerlandaises de Zoologie, 13, 265-343.

VREVEN, D., \& BLough, P. M. (1998). Searching for one or many targets: Effects of extended experience on the runs advantage. Journal of Experimental Psvchology: Animal Behavior Processes, 24, 98105.

(Manuscript received November 30, 1998; revision accepted for publication June 2, 1999.) 